\title{
Linear stability of channel entrance flow
}

\author{
Damien Biau \\ DIAM, University of Genova, Via Montallegro 1, 16145 Genova, Italy
}

\begin{abstract}
The spatial stability analysis of two dimensional, steady channel flow is investigated in the downstream entry zone. A model based on previous work, is presented for the base flow, which represents a small deformation of plane Poiseuille flow. The base flow evolution towards the fully developed state comes from the experimental and theoretical study of M. Asai and J.M. Floryan "Certain aspects of channel entrance flow", Phys. Fluids 16 (2004). This flow is found to be more stable than the parabolic Poiseuille flow, both when exponentially and algebraically growing disturbances are examined. The most destabilizing base flow defect is calculated using a variational method. The compromise between the destabilising effect of the defect, which diffuses downstream, and the instability growth is found to be insufficient to provoke transition in the downstream laminar flow.
\end{abstract}

Key words: Hydrodynamic instability, channel flow.

PACS: 47.15.Fe, 47.20.-k, 47.27.nd

\section{Introduction}

This work is concerned with the stability properties of entry channel flow. The disturbance growth mechanism in parallel and quasi-parallel flows is of great interest to understand transition to turbulence and has been the object of several theoretical and experimental studies.

For simple shear flow cases, such as the Poiseuille flow, the experimental flow behavior and the theoretical results are quite different. Transition to turbulence is observed for minimum transition Reynolds number around $R e=1000$ (Nishioka and Asai [12]), well below the critical value provided by linear stability theory, i.e. $R e_{c}=5772$ (Orszag [14]). The transition may occur at Reynolds number lower than 5772 because of the subcritical character of the instability. In fact, the stability characteristics of shear flows are extremely sensitive to external perturbations. This property, linked to the non-normal

Preprint submitted to Elsevier Science 10 October 2006 
nature of the dynamical disturbances operator, is crucial in determining the outcome of laboratory experiments.

Research on the topic of transition in shear flows in the last ten years has been concerned particularly with identifying worst case scenarios, i.e., those initial (or inlet) conditions responsible for the largest initial growth of disturbances, in a linearized setting. The disturbance can be distinguished by their growth: exponential or algebraic. The physical mechanisms describing the origin of disturbance growth may be extracted from the Reynolds-Orr equation. For parallel shear flows the source term for the instability can be positive in two manners. The phase shift at the wall implies a constructive interaction between the streamwise and wall normal velocity perturbation, at the origin of exponentially growing Tollmien-Schlichting waves. Alternatively, by the lift-up mechanism, wall normal fluctuation induces streamwise velocity fluctuation or streaks (Butler and Farrell [4], Trefethen et al. [17]). This lift-up mechanism implies algebraic amplification, which scales with the Reynolds number.

The argument goes that if the perturbation amplitude attains - even if only transiently - a sufficiently large value, some nonlinear boot-strapping effect will bring the system to transition. This new interpretation of linear stability theory has led many scientists studying transition in shear flows to almost abandon the traditional single-mode growth of the linear stability approach which captures the asymptotic behavior of the system, to pursue studies of nonmodal transient growth, optimal perturbations, and pseudospectra. The concept of three-dimensional optimal disturbances has been introduced by Butler and Farrell [4] and pursued by many others. All of the studies on optimals in wall-bounded shear flows (whether temporal or spatial) have shown that streamwise vortices transform into streaks downstream (in time or space) and that the disturbance energy, mostly carried by the streaks, can grow by orders of magnitudes over its initial value. The recent book by Schmid and Henningson [16] provides a complete account of the recent view of transition.

In this article we are interested on the influence of weak base flow distortions on linear stability results. The base flow uncertainty of the stability equation can be represented with $\delta U$, a possibly finite, but typically small, distortion of the idealized base flow $U_{\text {ref }}\left(\right.$ i.e.: $U=U_{\text {ref }}+\delta U$ ). The $\delta U$-pseudospectrum, introduced by Bottaro et al. [3], is defined as

$$
\Lambda_{\delta U}(L)=\left\{\alpha \in \mathbb{C}: \epsilon \in \Lambda\left(L\left(U_{\text {ref }}+\delta U\right)\right) \text {, for some } \delta U \text { with }\|\delta U\| \leq \epsilon\right\},
$$

where $\Lambda(L)$ is the spectrum of the linear stability operator $L$. The spectrum of $L(U+\delta U)$, is a subset of the unstructured pseudospectrum studied by Trefethen et al. [17]. It is well known that the pseudospectrum of a hydrodynamic stability operator can significantly differ from its spectrum when the operator is non-normal, indicating the strong sensitivity of non-normal operators to external excitations, and the consequences of this fact in hydrodynamic stability 
theory have been explored in details in a seminal paper by Trefethen et al. [17]. In particular, it has been shown that the pseudospectrum can protrude far into the unstable half plane beyond a critical value of the distortion amplitude, for nominally subcritical conditions. The most destabilising distortion, so-called the minimal defect, can be calculated by a variational approach. This problem has been recently addressed in the temporal setting by Bottaro et al. [3] for Couette flow, and in the spatial setting by Gavarini et al. [6] for pipe Poiseuille flow. The approach has been extended by Hwang and Choi [8] to the case of a two-dimensional wake, in order to suppress or enhance the absolute instability.

The main objective of the present work is to investigate the stability properties of two-dimensional, steady entry flow. The paper is organized as follows. The model of entry flow is described in section 2. The following sections are dedicated to the linear stability analysis, for both exponentially and algebraically growing disturbances, of realistic entry flow accruing from experiments by Asai and Floryan [1]. In the last section, before the conclusion, the worst possible entry flow is identified, i.e. the entry distortion, of fixed norm, that maximizes the growth rate of a mode, for subcritical values of the Reynolds number.

\section{Base flow defect formulation}

The channel flow induced by a streamwise pressure gradient is considered. The mean velocity is made dimensionless using the maximum velocity $U_{0}$, the distances scale with the half-channel height $h$, so that the base flow reads $U(y)=1-y^{2}$. In this section we present a model of the channel entry flow, downstream of the channel inlet, where the evolution has a universal character. Recent review of the relevant literature as well as a detailed description of the flow, in the case of channel entrance with sharp corners, are given in reference $[15]$.

We takes as a characteristic streamwise scale $L=h R e$, as in Prandtl's boundary layer approximation. This is the only difference with the model presented by Sadri and Floryan [15] and Asai and Floryan [1]. By proceeding as we do, the Reynolds number is scaled out in the dimensionless equations. From the physical point of view, the normal direction is characterized by diffusive effects and the longitudinal direction is dominated by advection, which justifies the Reynolds number in the ratio of the different characteristic lengths. By scaling the normal coordinates $y$ with $h$, the streamwise coordinate $x$ with $h R e$, and the streamwise velocity with $U_{0}$, it follows that $U_{0} / R e$ should be used as the scale for the normal velocity $V$. The pressure is normalized by $\rho\left(U_{0} / R e\right)^{2}$, with $\rho$ the density of the fluid. 
The dimensionless mean flow $(U, V, P)$, supposed to be two-dimensional and stationary, may be represented by:

$$
\begin{array}{rlrl}
U(x, y) & =U_{0}(y)+A U_{1}(y) e^{-\lambda\left(x-x_{0}\right)}, \\
V(x, y) & = & \lambda A V_{1}(y) e^{-\lambda\left(x-x_{0}\right)}, \\
-d P / d x & = & P_{0} & +A_{P} e^{-\lambda\left(x-x_{0}\right)} .
\end{array}
$$

The streamwise coordinate could be translated along $x$, i.e. we can introduce a fictious origin $x_{0}$. In the following $x_{0}$ is fixed to zero. The streamwise invariant solution $\left(U_{0}=1-y^{2}, P_{0}=2 / R e\right)$ corresponds to the fully developed Poiseuille flow. Asai and Floryan [1] give a relation between the velocity amplitude $A$ and the pressure amplitude as $A_{P}=-A / \operatorname{Re} d^{2} U_{1} /\left.d y^{2}\right|_{y=-1}$.

The flow is supposed to be slightly perturbed from plane Poiseuille flow, so the equation describing the dynamics of the disturbance is be linearized around the mean state $\left(U_{0}, P_{0}\right)$. This equation, for the streamfunction, takes the form of a generalized eigenvalues problem:

$$
D^{4} \psi+\lambda\left(U_{0} D^{2} \psi-U_{0}^{\prime \prime} \psi\right)=0
$$

associated with homogeneous Dirichlet and Neumann boundary conditions: $\psi=D \psi=0$ for $y= \pm 1$. The first two modes are $\lambda_{1}=-21.680 / R e$, $\lambda_{2}=-28.221 / R e$ and correspond, respectively, to sinuous (antisymmetric) and varicose (symmetric) modes, $c f$. figure 1.

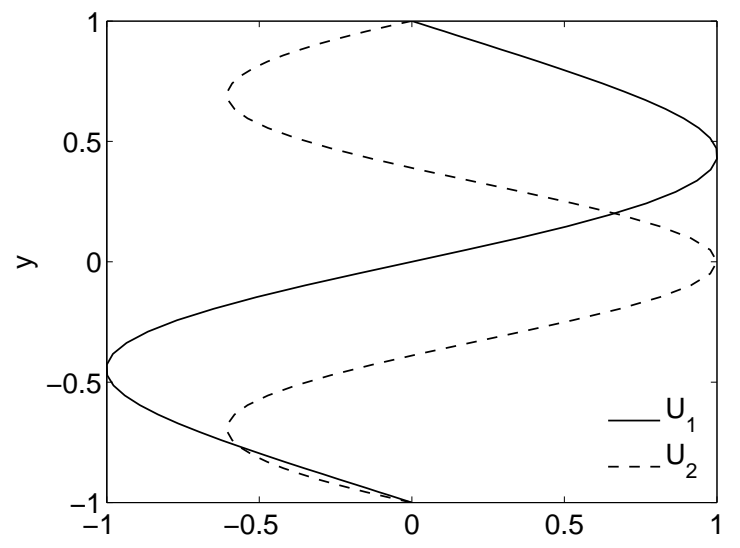

Fig. 1. The first and second eigenfunctions of equation (2), normalized with their maximum.

Any defect can be expressed as a weighted sum in the set of eigenmodes:

$$
\delta U(x, y)=\sum_{n}^{\infty} \kappa_{n} \tilde{U}_{n}(y) e^{-\lambda_{n}\left(x-x_{0}\right)} .
$$


The particular case $d P / d x=0$, corresponding to a sinous disturbance, leads to a Sturm-Liouville problem. In this form the projection weights $\kappa_{n}$ are simply determined by an appropriated scalar product; details are given in Appendix A.

\section{Linear stability of Asai and Floryan [1] experimental flow}

In the section we investigate the stability of the base flow found in the experimental and theoretical study of Asai and Floryan [1]. The contraction section used in the experiment is symmetric with respect to the midplane of the channel. So measurements show a deviation shape very similar to mode 2 in expansion (3) (i.e. $\kappa_{2} \gg \kappa_{n}$, for $n=1,3,4,5, .$. ).

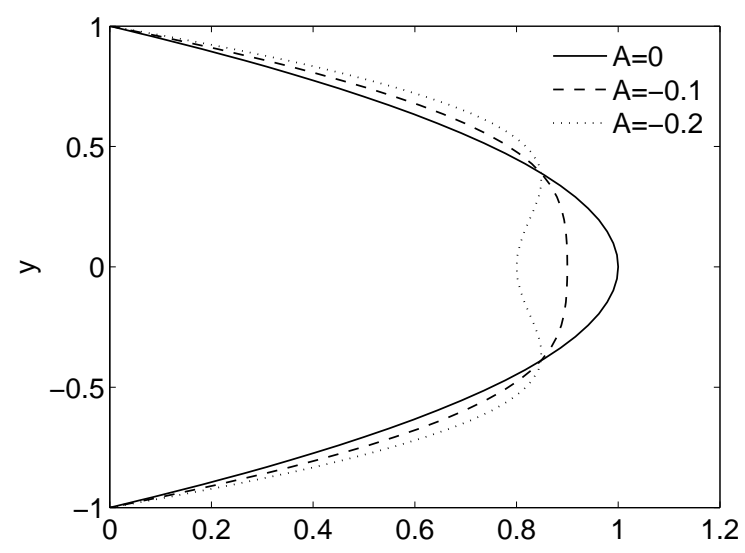

Fig. 2. Base flow profiles for different disturbance amplitudes.

\subsection{Modal analysis}

First, we study the evolution of disturbances with a modal analysis based on the parallel flow assumption. This classical stability analysis is focused on the sign of the least stable mode, labeled as Tollmien-Schlichting mode. The approach adopted here is spatial, implying that the eigenvalue problem is solved for $\alpha \in \mathbb{C}$ with $\omega$ and $\beta$ real. In compact form this system can be noted as $\mathcal{L} \tilde{q}_{n}=\alpha_{n} \tilde{q}_{n}$. The modal stability, for arbitrary base flow distortion, was studied earlier by Hidfi et al. [7]. The work of these authors has been conducted in the temporal framework, which is simpler but not as physically relevant as the spatial framework for the kind of open flows examined here. Although the neutral curve is independent of a spatial or temporal viewpoint, these two problems are quite different, because the spatial problem is elliptic. In fact, with the possible exception of the unstable mode, the upper half of 
the complex $\alpha$-plane contains downstream decaying modes while the lower half corresponds to upstream decaying modes. The spatial approach permits a direct comparison between the diffusion of the base flow and the growth of the unstable mode.

Using Squire theorem, the modal analysis can be reduced to the Orr-Sommerfeld equation with $\beta=0$ :

$$
\left\{\left(-i \omega+i \alpha U-\frac{1}{R e} \nabla^{2}\right) \nabla^{2}-i \alpha U^{\prime \prime}\right\} v=0
$$

The growth rate and corresponding phase velocity $c=\omega / \alpha_{r}$ contours, in the $(R e, A)$ plane, are displayed in figure 3.

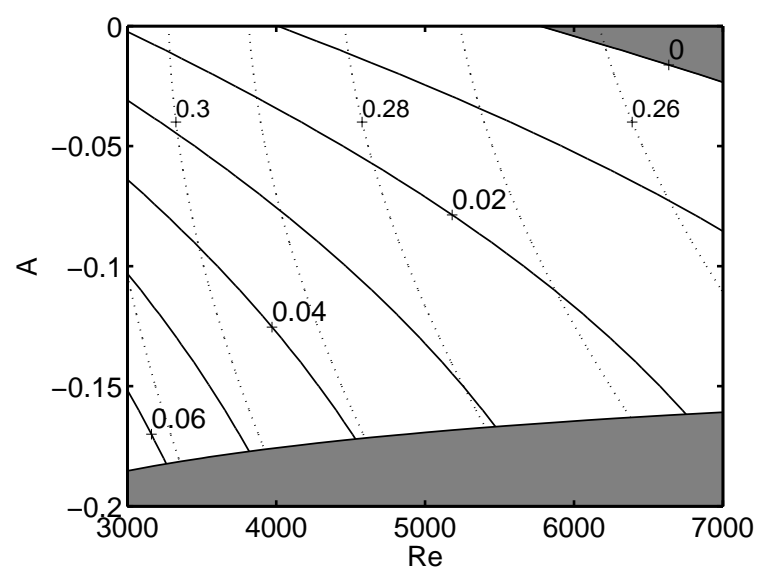

Fig. 3. Growth rate $\operatorname{imag}(\alpha)$ of TS mode (continuous lines) and corresponding phase velocity $c$ (dotted lines) in Reynolds number $R e$ versus the amplitude $A$ plane. The shaded regions are unstable.

The distortion on the entry flow displays a stabilizing effect on the viscous instability (Tollmien-Schlichting waves). For undisturbed Poiseuille flow, the critical Reynolds number is 5772. This threshold increases with the defect's amplitude. In addition, for high distortions an inviscid intability appears, linked to an inflection point of the mean velocity profile. This inviscid unstable mode is solution of Rayleigh's equation:

$$
\left\{(-i \omega+i \alpha U) \nabla^{2}-i \alpha U^{\prime \prime}\right\} v=0
$$

The growth rate and the corresponding phase velocity, for the inviscid instability, are presented in figure 4 . The eigenfunctions of the inviscid mode are centered near the half-channel line and the corresponding phase velocities are larger than for the viscous Tollmien-Schlichting modes. 


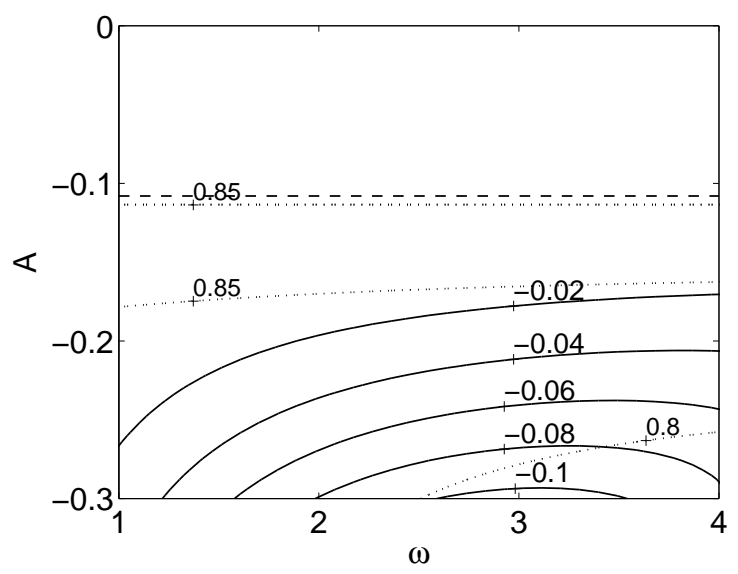

Fig. 4. Inviscid stability results. Growth rate $\operatorname{imag}(\alpha)$ (continuous lines) and corresponding phase velocity $c$ (dotted lines) in circular frequency $\omega$ versus the amplitude $A$ plane. The dashed line indicates the neutral curve for inviscid instability (i.e. $\operatorname{imag}(\alpha)=0)$.

\subsection{Transient growth}

As a next step, we investigate a transient, algebraically growing, perturbations, that cannot be attributed to single-mode exponential growth. The search for transiently growing perturbations starts with the search of optimal perturbation, i.e. the search of the inlet condition that provides the largest energy growth. Consequently, we define the maximum kinetic energy amplification $G_{\max }$ as,

$$
G_{\max }=\max _{\forall x \in[0 ; \infty[}\|q(x)\|_{E}
$$

with the normalization, $\|q(0)\|_{E}=1$. The subscript $E$ denotes an energetic norm defined as:

$$
\frac{G}{R e^{2}}=\frac{\left.\int_{-1}^{+1} u^{2} d y\right|_{x=x_{o p t}}}{\int_{-1}^{+1} v^{2}+\left.w^{2} d y\right|_{x=x_{0}}}
$$

The disturbances corresponding to this maximum take the form of streamwise elongated and steady structures. In this case the parallel base flow assumption is no longer valid and the characteristic scales for the perturbation must be identical to those for the base flow defined in section 2. The algebraically growing perturbations can be adequatly described by the use of linearized equations in which the long scale $(h R e)$ is used to normalize streamwise length, and the short scale $(h)$ is used for the cross-stream directions. Applying these scales to the linearized Navier-Stokes equations it follows that the disturbance equations, at leading order, are independent of the Reynolds number and parabolic in the streamwise direction. Moreover, the linearization permits a mode-by-mode study in Fourier space. Along the homogeneous directions the 
perturbations are expressed in Fourier-like form : $\exp (i \beta z-i \omega t)$. Finally, the disturbance equations take the symbolic form $A q_{x}=B q$, which in expanded form, read:

$$
\begin{aligned}
& u_{x}+v_{y}+i \beta w=0 \\
& -i \omega u+(U u)_{x}+V u_{y}+U_{y} v=u_{y y}-\beta^{2} u_{z z} \\
& -i \omega v+(V u+U v)_{x}+2(V v)_{y}+i \beta V w=-p_{y}+v_{y y}-\beta^{2} v_{z z} \\
& -i \omega w+(U w)_{x}+(V w)_{y}=-i \beta p+w_{y y}-\beta^{2} w_{z z}
\end{aligned}
$$

These equations are associated to homogeneous Dirichlet boundary conditions at the walls. This system was previously used by Luchini [11] for optimal perturbations in non-parallel boundary layer. The inflow condition for the equations (7) was computed using the discrete Lagrange multipliers method: a Lagrangian functional is defined as

$$
\mathcal{L}=G(L)+\int_{0}^{L} \bar{p}^{T}\left[\left(A q_{x}\right)-B q\right] d x
$$

maximization of $\mathcal{L}$ leads to the unconstrained set of equations:

$$
\begin{aligned}
& (A q)_{x}=B q \\
& p(x=L, y)=-2 H^{-1} A q(x=L, y) \\
& \bar{A}^{T} p_{x}=-\bar{B}^{T} p \\
& q(x=0, y)=-\left(2 E_{L}\right)^{-1} H^{-1} A p(x=0, y)
\end{aligned}
$$

The perturbations are normalized such that $E_{x=0}=1$. The equations are discretised using a Chebyshev pseudospectral method in the wall normal direction and a finite difference scheme in the streamwise direction. The iterations were pursued until the subsequent changes in the maximal gain dropped below a threshold value, fixed at $10^{-4}$. The parameters $\omega_{\text {opt }}, \beta_{\text {opt }}$ and $x_{\text {opt }}$, corresponding to the largest possible gain, at fixed Reynolds number and amplitude, are obtained using a shooting method. The optimal frequency was found to vanish in all cases. The maximum gain $G_{\text {max }} / R e^{2}$ and corresponding $\beta_{\text {opt }}$ and $x_{\text {opt }}$ are drawn in figure 5 as function of the initial defect amplitude.

The streaks presents a weak sensitivity to the mean flow distortion, in contrast with TS waves. When the defect amplitude increases, the streaks maximum amplitude decreases slowly. Simultaneously, the corresponding location decreases and the streaks' width increases. An example of optimal perturbation is presented in figure 6 . 

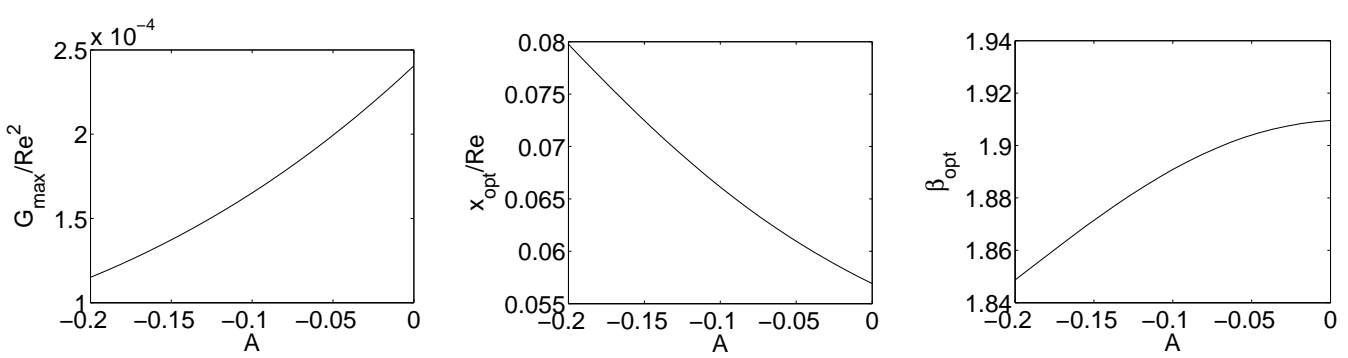

Fig. 5. Maximum gain $(G)$, optimal streamwise location $x_{\text {opt }}$ and optimal spanwise wave number $(\beta)$ versus defect amplitude $A$.
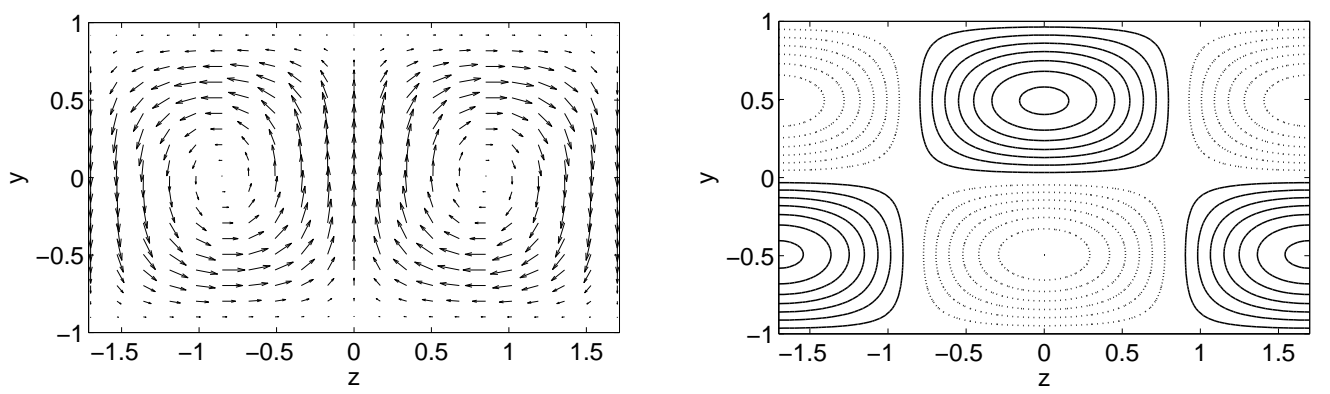

Fig. 6. Optimal inflow disturbances at $x=0$ (left) and resulting streaks at $x=x_{\text {opt }} / \operatorname{Re}$ (right) for $A=-0.2$. The inflow perturbation is represented through the cross-stream velocity (vectors). The outflow is displayed with isolines of the streamwise velocity; the positive and negative values are respectively denoted by continuous and dotted lines.

The optimal perturbations in the entry channel flow are very similar to those found for the unperturbed Poiseuille flow ( $c f$. Biau and Bottaro [2]).

\section{Worst-case scenario}

In the previous sections the stability properties of realistic entry flows was investigated. The results show a net stabilizing effect. Now we are interested in finding the worst, most destabilizing, flow distortion, following the method developed by Bottaro et al. [3]. With a temporal stability study Bottaro et al. described a technique to identify optimally configured defects of the base flow capable of rendering Couette flow linearly unstable. The work has been extended to the spatial frame by Gavarini et al. [6] for the case of pipe Poiseuille flow. This work has shown that the optimal base flow distortion is efficient to provoke transition in laminar pipe flow. Hwang and Choi [8] have investigated the effect of basic-flow modification on the absolute instability in a wake at low Reynolds number. Using the method of calculus of variation, they opti- 
mally modify the basic flow to suppress or enhance the absolute instability. For a two-dimensional parallel model wake and a circular-cylinder wake, this work shows that the positive and negative velocity perturbations to the basic flows, respectively, at the wake centreline and separating shear layer suppress the absolute instability.

Here, the same technique is used for entry channel flow in subcritical conditions, by considering disturbances developing in space. In this section, the stability analysis is focused on the modal study. As shown previously, the algebraically growing perturbations are weakly sensitive to small base flow deviation.

\subsection{The sensitivity functions}

Operators resulting from perturbations of the base flow only, unlike general perturbations, are subject to Squires theorem and transformation. Hence, we limit ourselves here to considering only the Orr-Sommerfeld equation. An infinitesimal, locally parallel variation $\delta U$ in the Poiseuille flow, injected into the Orr-Sommerfeld equation, symbolically written as $\mathcal{L}_{O S}$, leads to:

$$
\mathcal{L}_{O S}(U+\delta U ; \alpha+\delta \alpha)(\mathbf{v}+\delta \mathbf{v})=0
$$

which can be rewritten, after linearisation, as:

$$
\mathcal{L}_{O S} \delta \mathbf{v}+\delta U \frac{\partial \mathcal{L}_{O S}}{\partial U} \mathbf{v}+\delta \alpha \frac{\partial \mathcal{L}_{O S}}{\partial \alpha} \mathbf{v}=0
$$

In order to isolate the eigenvalue variation, we now project onto the adjoint subspace spanned by $a(y)$, with the scalar product $(\cdot, \cdot)$ defined by $(p, q)=\int_{y} p^{*} q d y$. The function $a(y)$ is solution of the adjoint Orr-Sommerfeld equation:

$$
\mathcal{L}_{O S}^{\dagger} \mathbf{a}=\left\{\left[-i \omega+i \alpha^{*} U+\frac{1}{R e} \nabla^{2}\right] \nabla^{2}+2 i \alpha^{*} U^{\prime} \partial_{y}\right\} \mathbf{a}=0
$$

with homogeneous Dirichlet and Neumann boundary conditions. The variation in a given eigenvalue arising from an arbitrary variation $\delta U$ is:

$$
\delta \alpha=\int_{-1}^{+1} G_{U} \delta U d y
$$


where the sensitivity function $G_{U}$ is an appropriate combination, after integration by parts, of direct and adjoint eigenfunctions of the given mode:

$$
G_{U}=\alpha \mathbf{a}^{*} \nabla^{2} \mathbf{v}-\alpha\left(\mathbf{a}^{*} \mathbf{v}\right)^{\prime \prime}
$$

with the direct-adjoint normalization:

$$
-\left(\mathbf{a}, \partial_{\alpha} L_{O S} \mathbf{v}\right)=\int_{-1}^{1} \mathbf{a}^{*}\left[U^{\prime \prime}+2 \alpha(\alpha U-\omega)+\left(\frac{4 i \alpha}{R e}-U\right) \nabla^{2}\right] \mathbf{v} d y=1
$$

\subsection{Distortions in the base flow: the minimal defects}

We define an energy-like norm to quantify the deviation from the undisturbed Poiseuille flow:

$$
\epsilon=\int_{y}\left(U(x=0, y)-U_{\text {ref }}(y)\right)^{2} d y .
$$

Using Lagrange multipliers and following the method described in Bottaro et al. [3], we obtain :

$$
\left\{\begin{array}{l}
U=U_{r e f}+\frac{\operatorname{Im}\left(G_{U}(x=0, y)\right)}{2 \lambda} \\
\lambda=-\sqrt{\frac{1}{4 \epsilon} \int_{y}\left(\operatorname{Im}\left(G_{U}(x=0, y)\right)\right)^{2} d y}
\end{array}\right.
$$

The most sensitive mode, which maximises the imaginary part of the sensitivity function $\|\operatorname{Im}(G u)\|_{\infty}$ with respect to $\omega$, is followed during the iterative process. The most sensitive mode is preferred to the most unstable one, because the latter could converge to a local minimum. The results are presented in figure 7, where the growth rate is represented in the plane Reynolds number, in the range $1000<R e<4000$, versus amplitude of the defect, in the range $5 \times 10^{-6}<\epsilon<10^{-4}$.

The domain of attraction of the laminar state shrinks for large $R e$ (as $R e^{\gamma}$ say, with $\gamma<0$ ), so that small but finite perturbations lead to transition. The minimum energy threshold satisfies the $R e^{\gamma}$-scalings, with $\gamma=-3 / 2$. Trefethen et al. [17], by using arguments based on dominant balance of nonnormal growth and nonlinear-feedback conjectured that $\gamma<-1$. Chapman [5], through a formal asymptotic analysis of the Navier-Stokes equations, found that for streamwise initial perturbations $\gamma=-3 / 2$ (factoring out the unstable modes), while for oblique initial perturbations $\gamma=-5 / 4$.

In order to compare the effect of the base flow defect diffusion against the growth of the instability, the so-called $e^{N}$ method is used. The case is consid- 


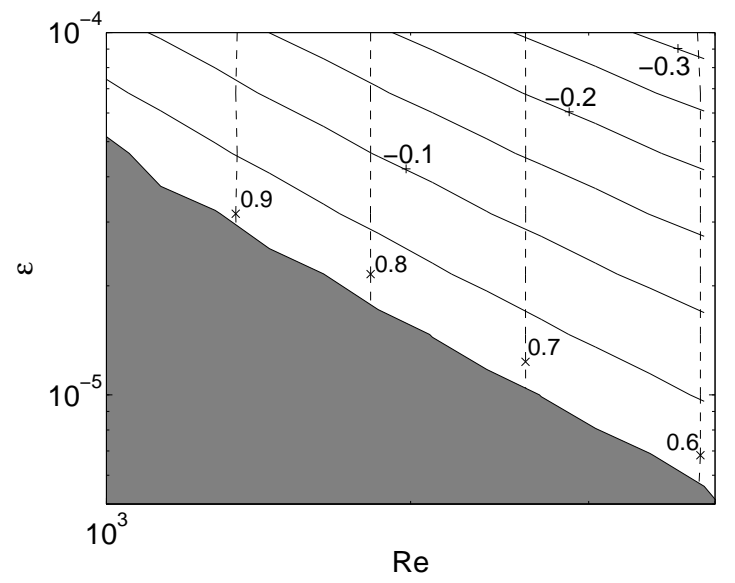

Fig. 7. Iso- $\alpha_{i}$ (continuous lines) in Reynolds number $(R e)$ and defect energy norm $(\epsilon)$ plane. The stable domain is shaded. The dashed lines are isolines of the frequency $\omega$ of the most unstable mode.

ered of $R e=2000$ and $\epsilon=5 \times 10^{-5}$; the corresponding optimal frequency is $\omega=0.773$.

The laminar basic flow is specified by projection on the set of eigenfunction (see equation 3), defined in section 2. The inflow distortion $\delta U(x=0, y)$ is obtained by the optimisation described previously. So that the mean velocity profiles are available at any streamwise location $(x)$ using the discrete projection:

$$
\delta U(x, y)=\sum_{n} \mathbb{Q}_{n}\left(\kappa_{n} e^{-\lambda_{n} x}\right) \quad \text { with } \quad \kappa=\mathbb{Q}^{-1} \delta U(x=0, y) .
$$

The matrix $\mathbb{Q}$ is constitued by the eigenfunctions of equation $(2)$, i.e. $\mathbb{Q}_{n m}=$ $\tilde{U}_{n}\left(y_{m}\right)$. Some shapes are plotted in figure 8 .

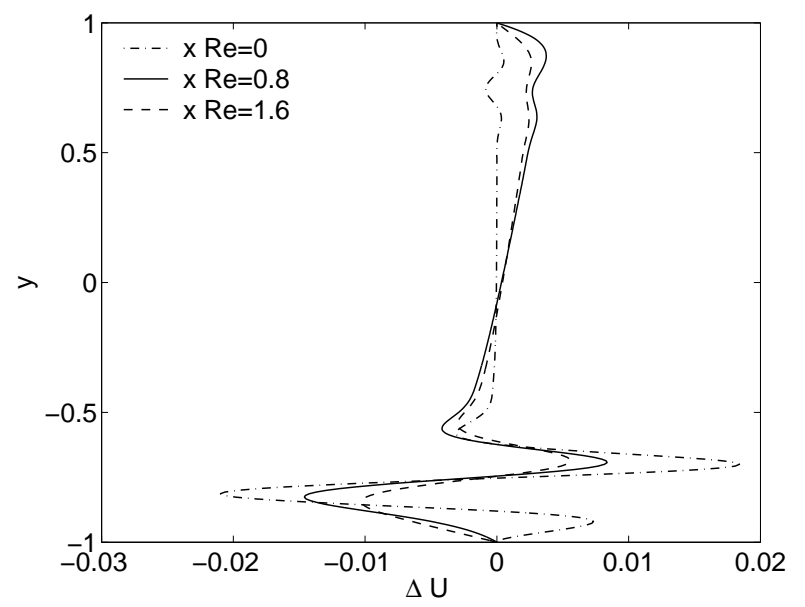

Fig. 8. Minimal defect for $R e=2000, \epsilon=5 \times 10^{-5}$ and $\omega=0.773$ at various dowstream locations.

A local stability analysis of this nearly parallel base flow is now performed 
to determine the growth rate of the locally unstable disturbances for various frequencies $\omega$. The stability diagram of amplified Tollmien-Schlichting waves as a function of the streamwise distance is depicted in figure 9.

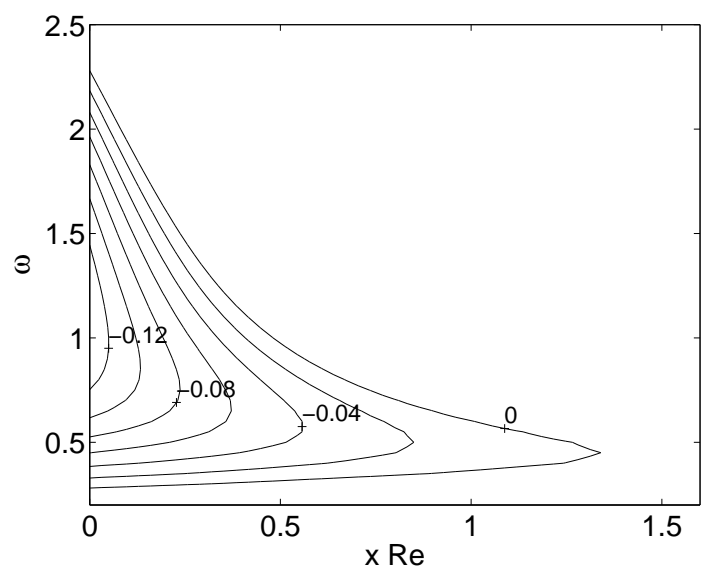

Fig. 9. Iso-growth rate $\left(\alpha_{i}\right)$ for $R e=2000, \epsilon=5 \times 10^{-5}$.

Let us consider now a wave which propagates downstream at a fixed frequency $\omega$. Figure 10 shows that this wave is amplified up to a certain distance and is damped further downstream because of the defect diffusing away. At any station $x>0$, the wave amplitude $A^{T S}$ can be related to its initial amplitude $A_{0}^{T S}$ by the relation : $A^{T S} / A_{0}^{T S}=\exp \left(\int_{0}^{x}-\alpha_{i} d x\right)$. The initial amplitude is linked to external noise through some receptivity mechanism. The streamwise variation of the natural logarithm $\ln \left(A^{T S} / A_{0}^{T S}\right)$ is plotted in figure 10 for several frequencies, the continuous line represents the envelope of these curves, called the $N$ factor: $N=\max \ln \left(A^{T S} / A_{0}^{T S}\right) ; \forall \omega$. At each location, $N$ represents the maximum amplification factor of the disturbances.

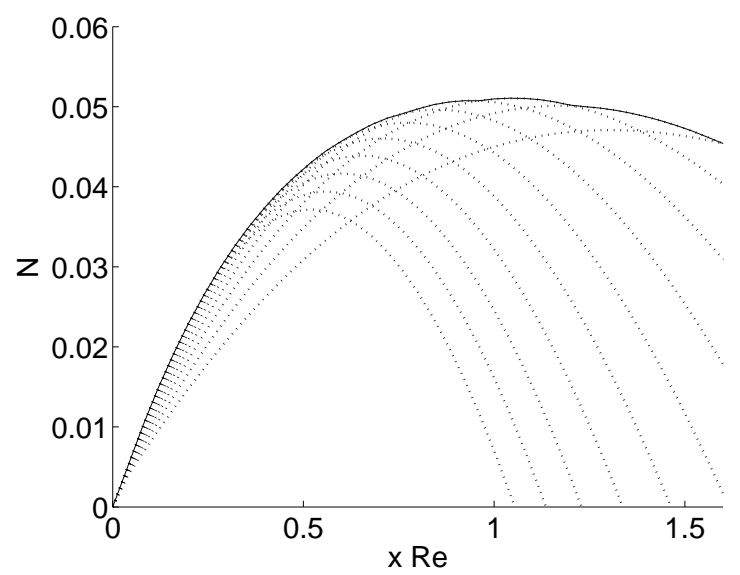

Fig. 10. $N$ factor for $R e=2000, \epsilon=5 \times 10^{-5}$ and $\omega=0.5,0.55, \ldots 1$.

Figure 10 illustrates the calculation of the $N$ factor. The associated evolution of mean flow defect si also represented. As can be seen, the amplification factor 
is too weak to overcome the viscous damping of the defect, and the $N$-factor maintains very small values.

\section{Conclusions}

In this study, spatial linear stability theory has been used to examine the stability of channel entrance flow. The presence of the entrance has a stabilizing effect in accordance with the well-know result that shear flows in a pressure drop region (accelerated flow) are more stable than those in the pressure decrease region (decelerated flow). For weak base flow distortion, in a negative pressure gradient (accelerated flow), the mean velocity profile has no inflection point and the instability - if it exists - is very weak. So the critical Reynolds number increases and the instability region decreases. For strong distortions, an inflection point gives rise to an inviscid instability described by Rayleigh theory. Secondly, the optimal distortion was determined but the instability growth is too weak to overcomes the viscous damping of the defect. Far downstream the defect is damped and critical values reach the classical results for Poiseuille flow.

Work in progress focuses on the stability of base flow distortions which depend on spanwise coordinate, to try and capture the defect of minimal norm which could cause transition in subcritical duct flow. The final goal will be to compare and link such steady, finite amplitude structures to experimental observations of streaks and self-sustained structures in channel flows.

Acknowledgements. The author wishes to thank professor A. Bottaro for his helpful comments on this work. The stay of the author at the University of Genova is supported by a Marie Curie grant through the FLUBIO project. 


\section{A Receptivity to inflow disturbances}

For boundary layer flows, Libby \& Fox [9] proposed a procedure to calculate the mode amplitude from an inflow condition imposed at $x_{0}$. Luchini [10] extended this results to overcome the leading edge singularity linked to algebraic form. In channel flow, without singularity at $x_{0}=0$, the Sturm-Liouville problem obtained for the auxiliary function $H=(\psi / U)^{\prime}$ is:

$$
\left(U^{3} H^{\prime}\right)^{\prime}+\left(3 U^{\prime \prime} U^{2}-\lambda U^{4}\right) H=0
$$

with the associated orthogonality relation :

$$
\int_{-1}^{1} H_{k} H_{l} d y=C_{k} \delta_{k l} \quad C_{k}=\int_{-1}^{1} H_{k}^{2} d y
$$

with $\delta_{k l}$ the kroneker symbol. The defect is expanded in the form:

$$
\delta \psi(x, y)=\sum_{k} A_{k} e^{\lambda\left(x-x_{0}\right.} \psi_{k}(y)
$$

so that the amplitude $A_{k}$ can be expressed using inflow condition:

$$
A_{k}=C_{k}^{-1} \int_{y}\left(\frac{\delta U\left(x_{0}, y\right)}{U(y)}\right)^{\prime}\left(\frac{\psi_{k}(y)}{U(y)}\right)^{\prime} U^{4} d y .
$$

Moreover, multiplying equation A.1 by $\psi_{k}$ and integrating shows that eigenvalues $\lambda$ are real and negatives, which implies the base flow distortion is damped in the downstream direction. 


\section{References}

[1] M. Asai and J.M. Floryan Certain aspects of channel entrance flow, Phys. Fluids, 16 (2004) 1160-1163.

[2] D. Biau and A. Bottaro Optimal perturbations and minimal defects: Initial paths of transition to turbulence in plane shear flows", Phys. Fluids, 16 (2004) 35153529 .

[3] A. Bottaro, P. Corbett and P. Luchini, The effect of base flow variation on flow stability, J. Fluid Mech. 476 (2003) 293-302.

[4] K.M. Butler and B.F. Farrell, Three-dimensional optimal perturbations in viscous shear flow, Phys. Fluids, 4 (1992) 1637-1650.

[5] S.J. Chapman, Subcritical transition in channel flows, J. Fluid Mech. 451 (2002) $35-97$.

[6] M.I. Gavarini, A. Bottaro and F.T.M. Nieuwstadt, The initial stage of transition in pipe flow: Role of optimal base flow distortions. J. Fluid Mech. 517 (2004) 131-165.

[7] A. Hidfi, M. O. Touhami and J.K. Naciri, Channel entrance flow and its linear stability. J. Stat. Mech. : Theor. Exp., $\mathbf{P 0 6 0 0 3 ~ ( J u n e ~ 1 9 9 6 ) . ~}$

[8] Y. Hwang and H. Choi, Control of absolute instability by basic-flow modification in a parallel wake at low Reynolds number, J. Fluid Mech. 560 (2006) 465-475.

[9] P.A. Libby and H. Fox, Some perturbation solutions in laminar boundary-layer theory. J. Fluid Mech. 17 (1964) 433-449.

[10] P. Luchini, Reynolds-number-independent instability of the boundary layer over a flat surface. J. Fluid Mech. 327 (1996) 101-115.

[11] P. Luchini, Reynolds-number-independent instability of the boundary layer over a flat surface: Optimal perturbations, J. Fluid Mech. 404 (2000) 289-309.

[12] M. Nishioka and M. Asai, Some observations of the subcritical transition in plane Poiseuille flow, J. Fluid Mech. 150 (1985) 441.

[13] M. Nishioka, S. Iida and Y. Ichikawa, An experimental investigation of the stability of plane Poiseuille flow, J. Fluid Mech. 72 (1971) 731.

[14] S.A. Orszag, Accurate solution of the Orr-Sommerfeld stability equation, $J$. Fluid Mech. 50 (1971) 689.

[15] R.M. Sadri and J.M. Floryan Entry flow in a channel, Comp. Fluids, 31 (2002) $133-157$.

[16] P.J. Schmid and D.S. Henningson Stability and transition in shear flows, Springer Verlag, New-York.

[17], L.N. Trefethen and A.E. Trefethen and S.C. Reddy and T.A Driscoll, Hydrodynamic stability without eigenvalues. Science, 261 (1993) 578-584. 